\title{
$O$ atendimento de enfermagem no serviço de emergência à mulher vítima de violência
}

\author{
Nursing care in the emergency service for women victims of violence \\ Atención de enfermería en el servicio de urgencias para mujeres víctimas de violencia
}

Recebido: 25/10/2021 | Revisado: 0/11/2021 | Aceito: 03/11/2021 | Publicado: 08/11/2021

\author{
Lígia D’arc Silva Rocha Prado \\ ORCID: https://orcid.org/0000-0001-9690-9953 \\ Universidade Federal Fluminense, Brasil \\ E-mail: enfaligiaprado@hotmail.com \\ Rose Mary Costa Rosa Andrade Silva \\ ORCID: https://orcid.org/0000-0002-6403-2349 \\ Universidade Federal Fluminense, Brasil \\ E-mail: roserosauff@gmail.com \\ Eliane Ramos Pereira \\ ORCID: https://orcid.org/0000-0002-6381-3979 \\ Universidade Federal Fluminense, Brasil \\ E-mail: elianeramos.uff@gmail.com \\ Sandra Conceição Ribeiro Chícharo \\ ORCID: https://orcid.org/0000-0002-1487-0088 \\ Universidade Federal Fluminense, Brasil \\ E-mail: sandracrchicharo@gmail.com \\ Marcia Bastos Couto \\ ORCID: https://orcid.org/0000-0003-0114-8691 \\ Universidade Federal Fluminense, Brasil \\ E-mail: marbcouto@gmail.com \\ Janaina Mengal Gomes Fabri \\ ORCID: https://orcid.org/0000-0002-4777-4746 \\ Universidade Federal Fluminense, Brasil \\ E-mail: janamgfabri@gmail.com \\ Isadora Pinto Flores \\ ORCID: https://orcid.org/0000-0002-5429-672X \\ Universidade Federal Fluminense, Brasil \\ E-mail: isadoraflores@ outlook.com
}

\section{Resumo}

Objetivo: analisar na literatura científica as estratégias de atendimento pelos profissionais de Enfermagem no Serviço de Emergência à mulher vítima de violência, trazendo debate. Método: revisão integrativa realizada na BVS, MEDLINE/PubMed, SciELO e CINAHL, em abril e maio de 2021. Resultados: a amostra foi composta por 17 artigos, percebendo-se cinco perspectivas horizontais: o cuidado do enfermeiro à mulher vítima de violência; reparo aos danos causados à saúde; os impactos sociais da violência contra a mulher; o aumento da violência contra a mulher na pandemia e, por fim, as estratégias de combate à violência contra a mulher. Conclusão: os estudos revelaram que o atendimento de Enfermagem se caracteriza pelas suas variadas funcionalidades que podem constituir ferramentas estratégicas para a saúde da mulher vítima de violência doméstica no contexto da saúde pública, evidenciando-se a necessidade da construção de equipes multidisciplinares.

Palavras-chave: Cuidados de enfermagem; Violência contra a mulher; Pandemia COVID-19; Atendimento de emergência.

\begin{abstract}
Objective: to identify in the scientific literature the care provided by Nursing professionals in the Emergency Service to women who are victims of violence. Method: integrative review, carried out in the VHL, MEDLINE/PubMed, SciELO and CINAHL databases, in April and May 2021. Results: from the sample of 17 articles, it was perceived five horizontal perspectives: the care provided by nurses to women victims of violence; repairing damage caused to health; the social impacts of violence against women; the increase in violence against women in the pandemic and, finally, strategies to combat violence against women. Conclusion: Nursing care is characterized by its various functionalities which can constitute strategic tools for the health of women victims of domestic violence in the context of public health, highlighting the need to build multidisciplinary teams.
\end{abstract}

Keywords: Nursing care; Violence against women; COVID-19 pandemic; Emergency care. 


\section{Resumen}

Objetivo: identificar en la literatura científica la atención que brindan los profesionales de Enfermería en el Servicio de Emergencias a las mujeres víctimas de violencia. Método: revisión integradora realizada en las bases de datos BVS, MEDLINE / PubMed, SciELO y CINAHL, en abril y mayo de 2021. Resultados: la muestra estuvo conformada por 17 artículos analizados, percibiéndose cinco perspectivas horizontales: la atención brindada por enfermeras a mujeres víctimas de violencia; reparar los daños causados a la salud; los impactos sociales de la violencia contra la mujer; el aumento de la violencia contra la mujer en la pandemia y, finalmente, las estrategias para combatir la violencia contra la mujer. Conclusión: La atención de enfermería se caracteriza por sus diversas funcionalidades que pueden constituir herramientas estratégicas para la salud de las mujeres víctimas de violencia intrafamiliar en el contexto de la salud pública, destacando la necesidad de construir equipos multidisciplinarios.

Palabras clave: Atención de enfermería; Violencia contra la mujer; Pandemia de COVID-19; Cuidados de emergencia; Revisión.

\section{Introdução}

Atualmente, a violência contra a mulher tem sido assunto extensamente debatido, sobretudo no período de pandemia da COVID-19, com registro do aumento de casos em todo o mundo (di Franco et al., 2020; Estadão, 2020; G1, 2020, 2020). A Enfermagem, em sua atuação no serviço de emergência, desempenha um papel fundamental. A perspectiva é de que as enfermeiras de emergência influenciam no cuidado prestado às mulheres expostas à violência por parceiro íntimo5-8. O cuidado com a vítima é viabilizado, primeiramente, pela equipe de Enfermagem e por meio da equipe multidisciplinar. Esse cuidado, descrito, também, como a relação entre enfermeiro-paciente, possui a finalidade de promover a saúde, prevenir, diagnosticar, tratar e reabilitar as doenças das quais podem sofrer (LemusI et al., 2016).

Na conjuntura pandêmica da COVID-19, a violência contra a mulher se complexifica dentro das fenomenologias sociais; a violência se distingue pelo sofrimento causado à vítima e pela total perversidade vislumbrada nas marcas deixadas para toda a vida. Esse fenômeno indica, então, que as mulheres são mais vulneráveis, geralmente afetadas pela perda de seu bem-estar, segurança, das possibilidades de educação e desenvolvimento pessoal. Os abusos praticados por parceiros vão além da agressão física; independentemente de qual seja a sua forma, a vítima passa a sofrer danos psicológicos - a principal marca impressa -, resultando em lembranças dos horrores e das crueldades vivenciadas na própria pele como resquícios (Acosta et al., 2017).

O profissional de Enfermagem possui suporte legal para garantir a intervenção nesses casos. A violência e suas diferentes interfaces deve ser tratada pelas políticas públicas e, inevitavelmente, está inserida na atmosfera social, com todas as implicações aviltadas pela crise pandêmica da COVID-19. Nesse caminho, este estudo se justifica para que haja construção de conhecimentos voltados para o atendimento de Enfermagem, compreendendo a realidade sobreposta a mulheres em situação de violência doméstica, mais suscetíveis a tais atos devido ao isolamento demandado pela atual pandemia.

Frente ao exposto, objetiva-se analisar trazendo ao debate, através da literatura adjacente publicada, as estratégias de atendimento de Enfermagem no Serviço de Emergência às mulheres vítimas de violência.

\section{Metodologia}

Revisão integrativa de literatura, na sequência de seis passos: 1) identificação do tema e elaboração da questão norteadora da pesquisa; 2) estabelecimento de critérios de inclusão e exclusão para a busca na literatura; 3) definição das informações a serem extraídas dos estudos encontrados; 4) avaliação dos estudos incluídos; 5) interpretação dos resultados; 6) apresentação da síntese realizada (Chícharo et al., 2021; Mendes et al., 2008).

Utilizou-se a estratégica PICO, com o mapeamento dos termos de acordo com o acrônimo: P (população) $=$ profissionais da Enfermagem; I (intervenção) = atuação/acolhimento no Serviço de Emergência; O (desfecho) = cuidado à mulher vítima de violência (C. M. da C. Santos et al., 2007). Não fora aplicado o critério C (comparação). Nesse caminho, 
elaborou-se a seguinte questão norteadora da pesquisa: "Como o profissional de Enfermagem atua no Serviço de Emergência, viabilizando o cuidado da mulher vítima de violência?".

A busca se deu em abril e maio de 2021, nas bases de dados Biblioteca Virtual em Saúde (BVS), Medical Literature Analysis and Retrieval System Online (MEDLINE) via US National Library of Medicine (PuBMed), Scientific Electronic Library Online (SciELO) e Cumulative Index to Nursing and Allied Health (CINAHL). Os descritores foram selecionados de acordo com os Descritores em Ciências da Saúde (DeCS) e Medical Subject Headings (MeSH), entrelaçados pelos operadores booleanos AND e OR, conforme quadro a seguir:

Quadro 1: Estratégias de busca nas bases de dados BVS, MEDLINE/PubMed, SciELO e CINAHL

\begin{tabular}{|c|c|}
\hline BASE DE DADOS & ESTRATÉGIA DE BUSCA \\
\hline BVS & 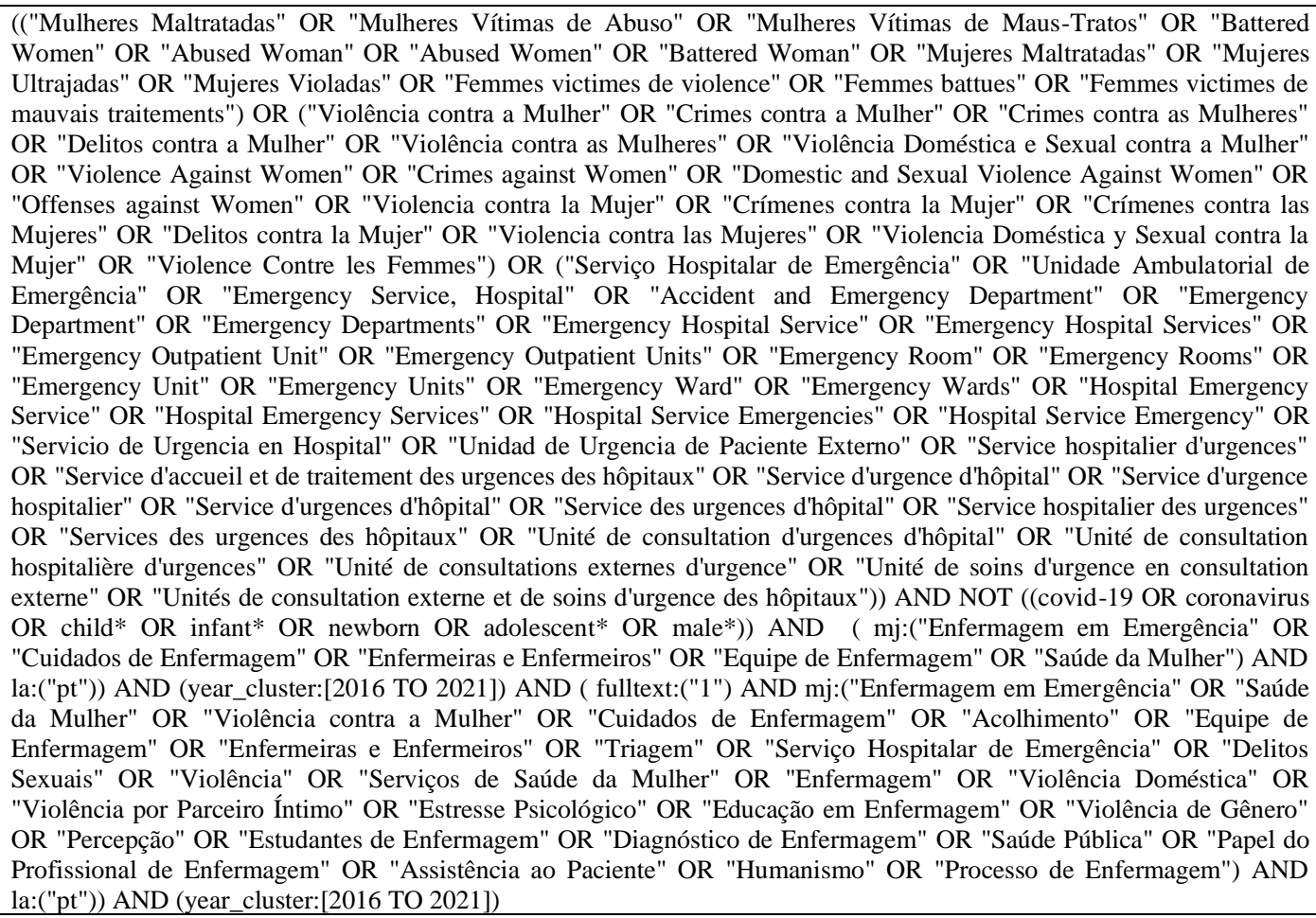 \\
\hline MED & 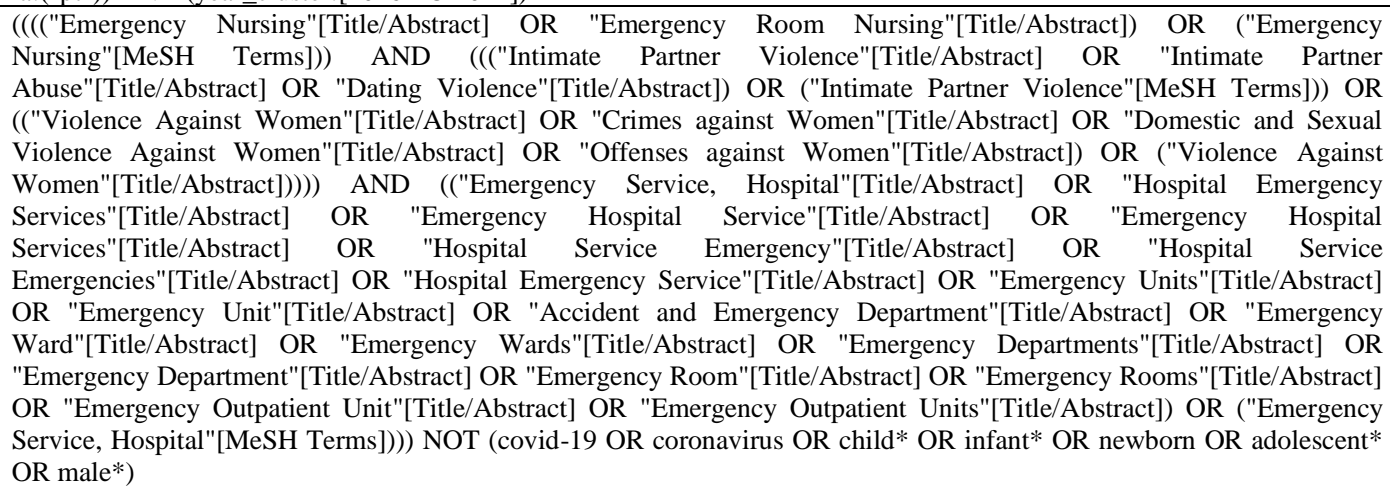 \\
\hline$S$ & $\begin{array}{l}\text { (("Mulheres Maltratadas" OR "Mulheres Vítimas de Abuso" OR "Mulheres Vítimas de Maus-Tratos" OR "Battered } \\
\text { Women" OR "Abused Woman" OR "Abused Women" OR "Battered Woman" OR "Mujeres Maltratadas" OR "Mujeres } \\
\text { Ultrajadas" OR "Mujeres Violadas" OR "Femmes victimes de violence" OR "Femmes battues" OR "Femmes victimes de } \\
\text { mauvais traitements") OR ("Violência contra a Mulher" OR "Crimes contra a Mulher" OR "Crimes contra as Mulheres" } \\
\text { OR "Delitos contra a Mulher" OR "Violência contra as Mulheres" OR "Violência Doméstica e Sexual contra a Mulher" } \\
\text { OR "Violence Against Women" OR "Crimes against Women" OR "Domestic and Sexual Violence Against Women" OR } \\
\text { "Offenses against Women" OR "Violencia contra la Mujer" OR "Crímenes contra la Mujer" OR "Crímenes contra las } \\
\text { Mujeres" OR "Delitos contra la Mujer" OR "Violencia contra las Mujeres" OR "Violencia Doméstica y Sexual contra la } \\
\text { Mujer" OR "Violence Contre les Femmes") OR ("Serviço Hospitalar de Emergência" OR "Unidade Ambulatorial de } \\
\text { Emergência" OR "Emergency Service, Hospital" OR "Accident and Emergency Department" OR "Emergency } \\
\text { Department" OR "Emergency Departments" OR "Emergency Hospital Service" OR "Emergency Hospital Services" OR } \\
\text { "Emergency Outpatient Unit" OR "Emergency Outpatient Units" OR "Emergency Room" OR "Emergency Rooms" OR } \\
\text { "Emergency Unit" OR "Emergency Units" OR "Emergency Ward" OR "Emergency Wards" OR "Hospital Emergency }\end{array}$ \\
\hline
\end{tabular}




\begin{tabular}{|c|c|}
\hline & $\begin{array}{l}\text { Service" OR "Hospital Emergency Services" OR "Hospital Service Emergencies" OR "Hospital Service Emergency" OR } \\
\text { "Servicio de Urgencia en Hospital" OR "Unidad de Urgencia de Paciente Externo" OR "Service hospitalier d'urgences" } \\
\text { OR "Service d'accueil et de traitement des urgences des hôpitaux" OR "Service d'urgence d'hôpital" OR "Service d'urgence } \\
\text { hospitalier" OR "Service d'urgences d'hôpital" OR "Service des urgences d'hôpital" OR "Service hospitalier des urgences" } \\
\text { OR "Services des urgences des hôpitaux" OR "Unité de consultation d'urgences d'hôpital" OR "Unité de consultation } \\
\text { hospitalière d'urgences" OR "Unité de consultations externes d'urgence" OR "Unité de soins d'urgence en consultation } \\
\text { externe" OR "Unités de consultation externe et de soins d'urgence des hôpitaux")) AND NOT ((covid-19 OR coronavirus } \\
\text { OR child* OR infant* OR newborn OR adolescent* OR male*)) AND ( mj:("Enfermagem em Emergência" OR "Cuidados } \\
\text { de Enfermagem" OR "Enfermeiras e Enfermeiros" OR "Equipe de Enfermagem" OR "Saúde da Mulher") AND la:("pt")) } \\
\text { AND (year_cluster:[2016 TO 2021]) }\end{array}$ \\
\hline CINAHL & $\begin{array}{l}\text { ("Intimate Partner Violence" OR "Intimate Partner Abuse" OR "Dating Violence" ) OR ( "Violence Against Women" OR } \\
\text { "Crimes against Women" OR "Domestic and Sexual Violence Against Women" OR "Offenses against Women" ) AND ( } \\
\text { "Emergency Service, Hospital" OR "Hospital Emergency Services" OR "Emergency Hospital Service" OR "Emergency } \\
\text { Hospital Services" OR "Hospital Service Emergency" OR "Hospital Service Emergencies" OR "Hospital Emergency } \\
\text { Service" OR "Emergency Units" OR "Emergency Unit" OR "Accident and Emergency Department" OR "Emergency } \\
\text { Ward" OR "Emergency Wards" OR "Emergency Departments" OR "Emergency Department" OR "Emergency Room" OR } \\
\text { "Emergency Rooms" OR "Emergency Outpatient Unit" OR "Emergency Outpatient Units" ) AND ( "Emergency Nursing" } \\
\text { OR "Emergency Room Nursing" ) NOT ( covid-19 OR coronavirus OR child* OR infant* OR newborn OR adolescent* } \\
\text { OR male*) }\end{array}$ \\
\hline
\end{tabular}

Fonte: Autores (2021).

Na revisão, incluíram-se artigos nos idiomas inglês, espanhol e português, que respondessem à questão norteadora estabelecida, no recorte temporal de 2016 a 2021. Foram excluídos os materiais encontrados que estavam em duplicata nas bases de dados. Após a utilização dos critérios estabelecidos, seguiu-se à leitura dos resumos dos artigos, para constatação de aproximação com a temática e, posteriormente, fora realizada a leitura na íntegra.

\section{Resultados e Discussão}

A busca inicial gerou um total de 164 artigos, sendo 110 da BVS, 17 da MEDLINE/PubMed e 37 da CINAHL. Na SciELO, não foram encontrados resultados. 147 foram encontrados em outras fontes, levando, assim, ao total de 311 artigos selecionados a princípio.

Após a aplicação dos critérios de inclusão e exclusão, chegou-se ao resultado abaixo, exposto na Figura 1. 
Figura 1: Fluxograma da estratégia de busca para a construção da revisão integrativa de literatura. Rio de Janeiro, RJ, Brasil, 2021
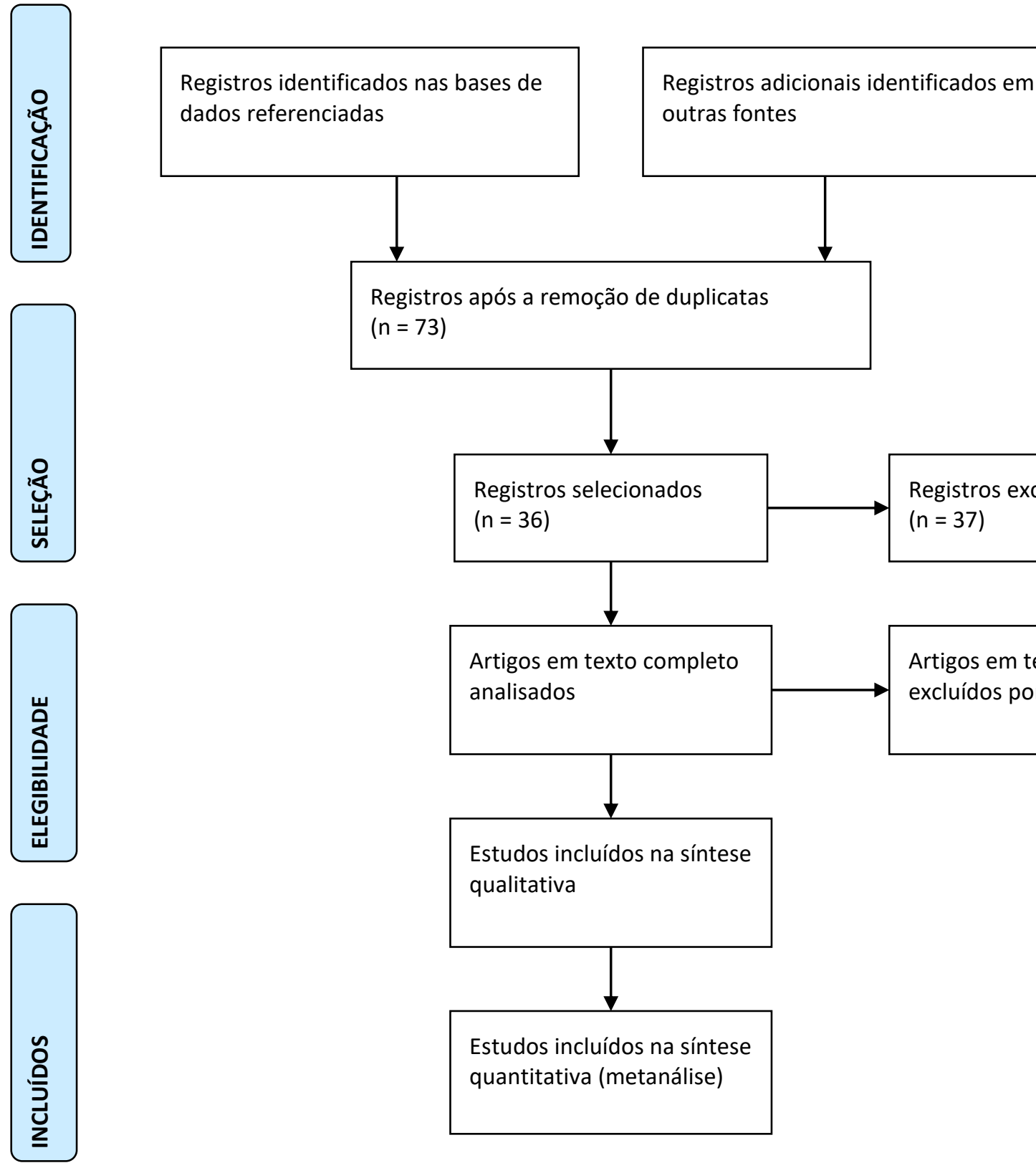

Registros adicionais identificados em outras fontes

$(n=73)$

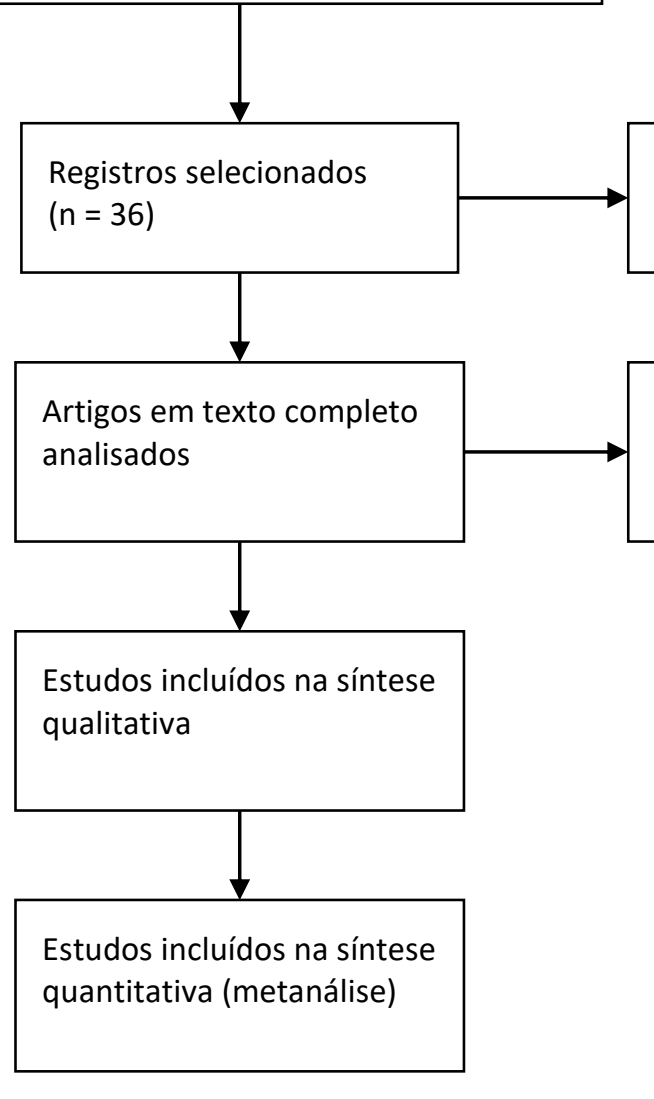

Registros excluídos $(n=37)$ Artigos em texto completo excluídos por razões

Fonte: Autores (2021).

Dentre os 17 estudos selecionados, analisados e citados, constam publicações que trazem questões que corroboram com o entendimento sobre o atendimento de Enfermagem no Serviço de Emergência à mulher vítima de violência doméstica, sendo 10 deles incluídos pela síntese qualitativa e, os outros sete, pela síntese quantitativa ou metanálise.

Após a leitura dos artigos na íntegra, foram selecionadas as publicações por temas que enfatizaram a violência contra a mulher e o atendimento do enfermeiro no Serviço de Emergência. Salienta-se a prioridade da revisão, amplamente incluída nas discussões teóricas, com o intuito de elevar os conhecimentos científicos, bem como compreender a demanda assistencial para mulheres vitimadas. Sabe-se que esta é uma realidade geradora de impactos negativos para a mulher e sua família, implicando em alterações no seu círculo social, trazendo, assim, reflexos para toda a sociedade, destacando-se a necessidade de atenção básica e o cuidado por parte dos serviços de saúde (Acosta et al., 2017). 


\section{Discussão}

Foram encontradas, na revisão realizada, cinco perspectivas horizontais, a saber:

1. O cuidado do enfermeiro à mulher vítima de violência: o serviço de Enfermagem, por vezes, é o contato inicial da vítima no atendimento e, por isso, o olhar atento e minucioso desses profissionais é de suma importância para a detecção do problema. A identificação dos sinais apresentados pela mulher na sua entrada, bem como a análise, a humanização e o cuidado com a coleta do histórico são fundamentais para que se apreenda, fidedignamente, o ocorrido (Acosta et al., 2017; Hewitt, 2015);

2. Reparo aos danos causados à saúde: a criação de uma equipe multidisciplinar é imprescindível para ações intersetoriais na promoção de projetos e campanhas que mostrem a essas mulheres que não estão sozinhas no enfrentamento das suas questões, e que haverá apoio nesse momento difícil de suas vidas, assim como a manutenção de um atendimento individualizado para cada uma delas. Essas campanhas poderão ser usadas para educar, conscientizar e reformular os valores de respeito e igualdade da sociedade atual.

3. Os impactos sociais da violência contra a mulher: o aumento do número de divórcios se configura como impacto social visível da experiência pandêmica. Há indícios de que há uma crescente nas estatísticas de separação, com crescimento de $15 \%$ no número de divórcios extrajudiciais, realizados diretamente em cartório, comparado ao ano anterior (Claudia, 2021).

4. O aumento da violência contra a mulher na pandemia: o registro de denúncias de violência contra a mulher no Brasil atingiu a marca de $105.821^{1}$. Esses números alarmantes correspondem a um aumento de $40 \%{ }^{2}$. A violência contra a mulher é um fenômeno mundial, ganhando força, sobretudo, no continente africano e na América Latina (di Franco et al., 2020; G1, 2020). Este breve panorama mostra que um importante passo foi dado em direção à necessidade de denunciar os casos de violência. No entanto, nenhuma política mais enérgica foi tomada, ou implementada uma nova política pública. A priori, o problema social da violência contra a mulher está sendo enfrentado por meio de campanhas contra esses abusos e com a ampliação dos canais de escuta e registro de denúncias.

5. As estratégias de combate à violência contra a mulher: é notável que os serviços de saúde têm um papel fundamental na detecção dos casos de violência, em tese por levar em consideração a abrangência de seu domínio sobre as famílias, pensando que, muitas vezes, o atendimento emergencial é a única ajuda procurada pela vítima. Uma melhor identificação e extensiva notificação dos casos de violência contra a mulher se fazem mandatórias para a criação de estratégias e planejamento de ações para a prevenção e detecção precoce, que se tornarão adequadas à realidade da área estudada (Freitas et al., 2018).

Diante desse cenário conflitante, há a necessidade de se discorrer acerca da violência, refletindo sobre a mulher que sofre em silêncio. Os relatos de enfermeiros apresentam, como diferencial nessa luta, as medidas protetivas contra o agressor, e que podem começar logo após o atendimento hospitalar. A mulher pode buscar assistência policial e judicial para garantir a recuperação da saúde física e emocional (Y. E. M. Lamadrid \& Gibert, 2016). Todo o processo de atendimento deve considerar, como fator principal, o resgate da dignidade da mulher como uma questão humanitária e de fundamental importância, sabendo que a violência deixa marcas físicas, psicológicas e emocionais, traumatizantes e indeléveis, seguidas por sentimentos de vulnerabilidade e incapacidade, levando a vítima à uma atitude de isolamento. Se este acontece, há maiores chances de perpetuação da violência, sendo realizada a manutenção dos atos cometidos pelo agressor. É exatamente nesse primeiro atendimento da Enfermagem que a mulher precisa lutar para enfrentar essa insegurança e vencer as dificuldades e o medo, ou seja, buscar a ajuda de outros profissionais para sanar o problema (Y. E. M. Lamadrid \& Gibert, 2016). 
A escuta qualificada é uma valiosa ferramenta para a vítima. Além disso, a mulher necessita se sentir acolhida e ficar à vontade para expressar seus medos e desejos, bem como seus traumas e ansiedades (Marshall et al., 2019). Todavia, é especificamente durante o primeiro atendimento realizado pela Enfermagem que a mulher começa a ser ouvida. Em decorrência da violência sofrida, toda informação é válida no momento de traçar o perfil do agressor. Por isso, espera-se que haja trocas de informações coerentes e éticas, e que o atendimento ocorra da melhor forma possível. Preferencialmente, deve ser realizado por meio de equipe multidisciplinar disponível no Serviço de Emergência da rede pública. É de grande relevância que haja compreensão mútua e disponibilidade entre os profissionais de saúde para a prevenção e manejo das situações de risco que a mulher vítima de violência enfrenta. Destarte, ela precisa se sentir amparada antes de expor suas demandas (W. J. dos Santos et al., 2018).

$\mathrm{O}$ atendimento é desafiador para o profissional de Enfermagem, primeiramente, pela demanda de pacientes que necessitam de cuidados e que são recebidos nos Serviços de Emergência

diariamente. Segundo, porque a mulher vítima de violência requer cuidados específicos, como acompanhamento médico e, também, de outros profissionais, dependendo do caso apresentado, dentre outros indicadores que podem atravessar o referido atendimento. Destaca-se que, por meio da "Notificação Compulsória", implementada em 2003, fora permitido informar aos gestores de saúde, justiça, cidadania e de ação social sobre mulheres violentadas atendidas nas redes de saúde pública ou privada (Netto et al., 2018).

A notificação de violência interpessoal/autoprovocada é o método a ser utilizado pelos profissionais que garante o registro dos casos e evidencia a situação de violência, o que favorece a perspectiva de políticas de enfrentamento, fortalecendo o combate.

Pesquisadores afirmam a importância da interdisciplinaridade e intersetorialidade, bem como a apropriação de novos saberes para a integralidade dos cuidados (Miranda et al., 2021).

Dentre as orientações e direcionamentos, o profissional procura amenizar o sofrimento dessas mulheres. Nos serviços de emergência, os enfermeiros estão diretamente envolvidos no gerenciamento e no cuidado, articulados para prestar apoio à mulher ${ }^{20}$. O atendimento ocorre no Serviço de Emergência e nas Unidades de Saúde básica, onde a escuta é efetuada (Y. E. M. Lamadrid \& Gibert, 2016).

O cuidado humanizado consiste em saber ouvir a mulher, atuando como testemunha de seu sofrimento, dando-lhe o direito à voz para que abandone o lugar de vítima, na tentativa de reverter a situação de angústia. Nesses casos, os enfermeiros mostram disponibilidade e aceitação para ouvi-la, estimulando a sua expressão do vivido, abrindo espaço para que assuma, como sujeito, a postura de protagonista do próprio cuidado (W. J. dos Santos et al., 2018).

Cabe ressaltar que o combate à violência é um trabalho coletivo para os programas e políticas que são voltadas para a atenção à violência contra a mulher e projetam medidas que proporcionem um bom acolhimento, uma assistência de qualidade e o direcionamento dessas vítimas aos outros procedimentos cabíveis (Miranda et al., 2021). Nenhuma forma de violência deve ser ignorada e, de nenhuma maneira, deve-se insinuar ou apontar a responsabilidade ou culpabilidade para a vítima.

Nesse contexto, é fundamental preservar os direitos da mulher, e isto envolve um trabalho multidimensional. Reiterase que a violência é um problema social e de saúde pública, que atinge e coloca em risco o desenvolvimento da população, independentemente da educação, raça, idade ou condição social (Netto et al., 2018).

Em escala, de uma a três mulheres expressam ter sofrido agressões físicas de seus parceiros, e uma a cada cinco mulheres é vítima de violência sexual ao longo da vida, o que configura uma relação desigual de gênero, podendo adquirir proporções epidêmicas (Paula \& Oliveira, 2019).

A Enfermagem, ao tratar do seu objeto - o cuidado -, atua em diversas áreas, cada uma com suas próprias especificidades e riscos (Rodrigues et al., 2020; A. G. Santos et al., 2017). O atendimento dessa disciplina tem um papel 
fundamental no tratamento dado às mulheres vítimas de violência doméstica, sabendo que essa violência é um impulso de agressividade que se embasa no modelo estruturador das relações de gênero, transformando-as em relações de poder, levando à usurpação do corpo do outro (W. J. dos Santos et al., 2018). Nesse sentido, o atendimento emergencial se configura como todo o cuidado e o apoio que a vítima precisa, tendo em vista sua vulnerabilidade física, psicológica e emocional. Ao colocar o cuidado como centro de sua prática, é possível, para a Enfermagem, que seja adquirida plena autonomia e que seja interpretada como uma esfera privilegiada na área da saúde (van der Wath, 2019). Como profissão da área da saúde, historicamente, ela tem o seu conhecimento direcionado para esse cuidado humano, considerando questões diversas do contexto individual de cada paciente (Paula \& Oliveira, 2019).

Ressalta-se que a principal limitação para a realização da presente revisão foram os dados oficiais referentes ao atendimento de Enfermagem para a assimilação clara da quantidade exata de mulheres atendidas no Serviço de Emergência. Embora a própria realidade denuncie o aumento da violência, são poucos os dados publicados no que diz respeito a esse atendimento no Serviço de Emergência e às falhas que ocorrem por consequência do período de pandemia da COVID-19. Aponta-se a ausência de mais pesquisas que delimitam a questão da violência nesse momento pandêmico junto ao atendimento de Enfermagem, como temáticas transversais delineadas por outros fatores que poderiam contextualizar novos estudos, além de abordar o cuidado desses profissionais às mulheres atendidas nesse serviço.

Os debates produzidos acerca da temática contribuem, diretamente, com a atenção e os cuidados básicos à vítima. Destaca-se que o profissional de Enfermagem vem contribuindo para a melhoria da saúde pública, trazendo em seu atendimento ferramentas de intervenção à violência contra a mulher. É possível afirmar que este profissional tem suporte legal para garantir a intervenção, respaldando-se nos princípios da "Política Nacional de Enfrentamento da violência contra a mulher", que propõe um trabalho articulado em rede (Secretaria Nacional de Enfrentamento à Violência contra as Mulheres, 2011).

A contribuição efetiva deste estudo, portanto, vislumbra o atendimento de Enfermagem como instrumento centrado no cuidado à mulher vítima de violência $\mathrm{e}$, dentre as estratégias, insere-se o atendimento no Serviço de Emergência. $\mathrm{O}$ profissional, ao se deparar com a demanda, deve sustentar um acolhimento sensível, lançando mão da sua escuta e da sua presença, não somente física, mas como um todo. $\mathrm{O}$ cuidado afetivo possibilita o estabelecimento de um vínculo forte $\mathrm{e}$ satisfatório para ambos da relação (Miranda et al., 2021; Secretaria Nacional de Enfrentamento à Violência contra as Mulheres, 2011). Reitera-se que a violência doméstica deve ser tratada de maneira multidimensional, pensando na conjuntura da saúde e das políticas públicas e, inevitavelmente, está inserida na atmosfera social, com todas as implicações aviltadas pela crise da COVID-19.

\section{Conclusão}

O apoio de enfermeiros próximos à mulher vítima de violência se torna imprescindível no Serviço de Emergência. Percebe-se o cuidado da Enfermagem como estratégia eficaz de combate, pensando na saúde da família e na integridade da vítima, preservada na sequência de atendimentos no serviço emergencial e pós-emergencial e, por isso, implica em um trabalho múltiplo nesse coletivo.

A investigação mostrou os desdobramentos da violência, apontando para os danos físicos, psicológicos e emocionais causados à saúde da mulher, bem como a afetação do psicológico e do emocional. Inclui-se, na temática da violência contra a mulher, a relevância das políticas públicas, trabalhando para a conscientização da população.

Nos últimos tempos, os debates teóricos acerca da violência contra a mulher vêm se ampliando, servindo como uma ferramenta potente de enfrentamento a esse mal enraizado e normalizado socialmente. O reconhecimento e apoio dos profissionais de Enfermagem se configuram como base fundamental para reverter o quadro de violência na vida da mulher.. 


\section{Referências}

Acosta, D. F., Gomes, V. L. de O., Oliveira, D. C. de, Gomes, G. C., \& Fonseca, A. D. da. (2017). Aspectos éticos e legais no cuidado de enfermagem às vítimas de violência doméstica. Texto \& Contexto - Enfermagem, 26(3). https://doi.org/10.1590/0104-07072017006770015

G1. (2020). Pandemia de Covid-19 fez violência contra a mulher disparar em todo o mundo. G1. https://oglobo.globo.com/celina/pandemia-de-covid-19-fezviolencia-contra-mulher-disparar-em-todo-mundo

Chícharo, S. C. R., Silva, R. M. C. R. A., Pereira, E. R., Nogueira, L. R. D., Prado, L. D. S. R., Silva, M. R. B. da, \& Vianna, T. A. (2021). Simulação realística no ensino de práticas de enfermagem em cuidados as feridas: Relato de experiências. Research, Society and Development, 10(9), e29010918180. https://doi.org/10.33448/rsd-v10i9.18180

Claudia. (2021). Pandemia expõe falhas nas relações amorosas e cresce o número de divórcios. https://claudia.abril.com.br/amor-e-sexo/pandemia-casaisdivorcio/

di Franco, M., Martines, G. F., Carpinteri, G., Trovato, G., \& Catalano, D. (2020). Domestic violence detection amid the COVID-19 pandemic: the value of the WHO questionnaire in emergency medicine. QJM: An International Journal of Medicine. https://doi.org/10.1093/qjmed/hcaa333

Freitas, R. J. M. de, Sousa, V. B. de, Costa, T. da S. C. e, Feitosa, R. M. M., Monteiro, A. R. M., \& Moura, N. A. de. (2018). Atuação dos enfermeiros na identificação e notificação dos casos de violência contra a mulher. HU Revista, 43(2), 91-97. https://doi.org/10.34019/1982-8047.2017.v43.2585

Hewitt, L. N. (2015). Intimate Partner Violence. Critical Care Nursing Clinics of North America, 27(2), 271-275. https://doi.org/10.1016/j.cnc.2015.02.004

Lamadrid, Y. E. M., \& Gibert, M. del P. (2016). Relación enfermera-paciente: una perspectiva desde las teorías de las relaciones interpersonales. Revista Cubana de Enfermeria, 32(4), e. http://www.revenfermeria.sld.cu/index.php/enf/article/view/976/215

LemusI, O. R. M., AcostaII, Y. A., \& DamasII, L. B. (2016). Violencia intrafamiliar contra la mujer. Revista Cubana de Enfermeria, 32(1), e. http://www.revenfermeria.sld.cu/index.php/enf/article/view/512/153

Marshall, A., Schultz, T., \& de Crespigny, C. (2019). Perceived changes and perceived transferability for treating intoxicated women victims of violence among emergency clinicians after an alcohol and other drugs training program. Drug and Alcohol Review, 38(5), 530-536. https://doi.org/10.1111/dar.12942

Mendes, K. D. S., Silveira, R. C. de C. P., \& Galvão, C. M. (2008). Revisão integrativa: método de pesquisa para a incorporação de evidências na saúde e na enfermagem. Texto \& Contexto - Enfermagem, 17(4), 758-764. https://doi.org/10.1590/S0104-07072008000400018

Miranda, A. P. C. de, González, A. M. dos S. R. de, Fraga, E., \& Dittz, E. da S. (2021). Violence against women: perceptions of maternity health professionals. Reme Revista Mineira de Enfermagem, 25, e-1390. https://doi.org/10.5935/1415.2762.20210038

Netto, L. de A., Pereira, E. R., Tavares, J. M. A. B., Ferreira, D. de C., \& Broca, P. V. (2018). Nursing performance in the conservation of women's health in situations of violence. REME: Revista Mineira de Enfermagem, 22. https://doi.org/10.5935/1415-2762.20180080

Paula, S. S. D. E., \& Oliveira, E. C. D. E. (2019). a Importância Da Atuação Do Enfermeiro Às Vítimas De Violência Sexual the Importance of the Nurse' S Activity To Victims of Sexual Violence. Revista Jurídica Uniandrade, 30(1), e. https://revista.uniandrade.br/index.php/juridica/article/view/1242

G1. (2020). Com restrições da pandemia, aumento da violência contra a mulher é fenômeno mundial. G1. https://g1.globo.com/mundo/noticia/2020/11/23/com-restricoes-da-pandemia-aumento-da-violencia-contra-a-mulher-e-fenomeno-mundial.ghtml

Rodrigues, C. G. S., Machado, J. C., Silva, L. R. da, Rodrigues, V. P., \& Pires, V. M. M. M. (2020). Rede de atenção à mulher em situação de violência doméstica sob a ótica da enfermeira. Research, Society and Development, 9(7), e656974676. https://doi.org/10.33448/rsd-v9i7.4676

Santos, A. G., Monteiro, C. F., Nunes, B. M. V. T., Benício, C. D. A. v., \& Nogueira, L. T. (2017). The nursing care analyzed according the essence of the care of Martin Heidegger. Rev. Cuba. Enferm, 33(3), e. http://www.revenfermeria.sld.cu/index.php/enf/article/view/1529/295.

Santos, C. M. da C., Pimenta, C. A. de M., \& Nobre, M. R. C. (2007). The PICO strategy for the research question construction and evidence search. Revista Latino-Americana de Enfermagem, 15(3), 508-511. https://doi.org/10.1590/S0104-11692007000300023

Santos, W. J. dos, Oliveira, P. P. de, Viegas, S. M. da F., Ramos, T. M., Policarpo, A. G., \& Silveira, E. A. A. da. (2018). Domestic Violence Against Women Perpetrated by Intimate Partner: Professionals' Social Representations in Primary Health Care / Violência Doméstica Contra a Mulher Perpetrada por Parceiro Íntimo: Representações Sociais de Profissionais da Atenção Primária. Revista de Pesquisa Cuidado é Fundamental Online, 10(3), 770-777. https://doi.org/10.9789/2175-5361.2018.v10i3.770-777

Secretaria Nacional de Enfrentamento à Violência contra as Mulheres. (2011). Política Nacional de Enfrentamento à Violência Contra as Mulheres.

Van Der Wath, A. (2019). Women exposed to intimate partner violence: a Foucauldian discourse analysis of South African emergency nurses' perceptions. African Health Sciences, 19(2), 1849. https://doi.org/10.4314/ahs.v19i2.7

Estadão. (2020). Violência contra a mulher aumenta em meio à pandemia; denúncias ao 180 sobem $40 \%$. 